Article

\title{
Promising Fungicides from Allelochemicals: Synthesis of Umbelliferone Derivatives and Their Structure-Activity Relationships
}

\author{
Le Pan ${ }^{1, *(\mathbb{D}}$, Dongyu Lei ${ }^{2}$, Lu Jin ${ }^{1, *}$, Yuan $\mathrm{He}^{1}$ and Qingqing Yang ${ }^{1}$ \\ 1 Chemical Engineering College, Xinjiang Agricultural University, Urumqi 830052, China; \\ hy941813@163.com (Y.H.); yqq1509914@sina.com (Q.Y.) \\ 2 Department of Physiology, Preclinical School, Xinjiang Medical University, Urumqi 830011, China; \\ leidongyu0109@163.com \\ * Correspondence: inmail911@sina.com (L.P.); chemkimlu@xjtu.edu.cn (L.J.); \\ Tel.: +86-180-992-783-61 (L.P.); +86-189-099-263-89 (L.J.)
}

Academic Editor: Jean-Marc Sabatier

Received: 20 October 2018; Accepted: 12 November 2018; Published: 16 November 2018

\begin{abstract}
Umbelliferone was discovered to be an important allelochemical in our previous study, but the contribution of its activity and structure has not yet been revealed. In this study, a series of analogues were synthesized to determine the skeleton of umbelliferone and examine its fungicidal activity. Furthermore, targeted modifications were conducted with three plant parasitic fungi to examine the lead compounds. Among those tested, compounds $\mathbf{2 f}$ and $\mathbf{1 0}$ were found to show excellent antifungal activity with an inhibitory rate over $80 \%$ at $100 \mathrm{ug} / \mathrm{mL}$. The study proves that umbelliferone can be a promising skeleton for fungicides discovery. In addition, the primary structure-activity relationship provides a good guidance for the discovery of novel fungicides based on natural products in the future.
\end{abstract}

Keywords: allelochemical; umbelliferone derivatives; antifungal activity; $\mathrm{EC}_{50}$ values

\section{Introduction}

Plant pathogenic fungi have jeopardized crop yields and food security globally. Fungicides have always been an efficient way of minimizing the cost of plant diseases [1]. However, the widespread use of traditional fungicides has led to increased resistance, mammalian toxicity, and a threat to the environment. This has stimulated the demand for integrated control measures with more selective, lower dose agents that have improved resistance [2-5]. Natural products (NPs) have been a rich source of novel fungicide development either with direct application or serving as a model for advanced modification [6]. However, with the explosive growth in NPs, it has become increasingly more difficult to pick the appropriate product from such a large range of compounds, and the introduction of novel molecules to the market has recently declined. Therefore, there is an urgent need for cost-effective tactics, instead of "blind search", in the development of new fungicides [7].

In nature, allelochemicals play an important role in plant ecological competition by inhibiting or enhancing the growth of the target organism. Allelochemicals have therefore been proposed as a promising means in the discovery of growth regulators and agrochemicals [8-10]. Coumarins are widely distributed in plants, and they have been proven to play a key role in plant allelopathic effect [11,12]. In our previous study, umbelliferone was identified as an important allelochemical of Stellera chamaejasme, and its analogs were synthesized to investigate antifungal activity. The structure-activity relationship (SAR) indicated that the modification of umbelliferone at the C7 hydroxy not only enhanced fungicidal activity but also decreased phytotoxicity [13]. In light of 
the above findings, coumarin derivatives were proposed to be an optimal skeleton (Figure 1). In this study, a series of compounds were designed to confirm the possible application of umbelliferone, and C7-substituted umbelliferones derivatives (UDs) were designed and synthesized to investigate their fungicidal activity against three devastating fungi: Alternaria alternata, Botrytis cinerea, and Fusarium oxysporum.

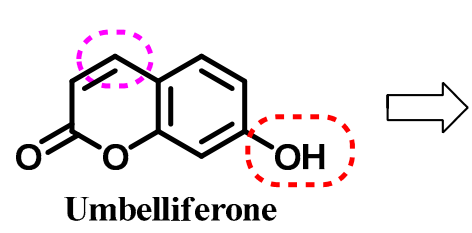

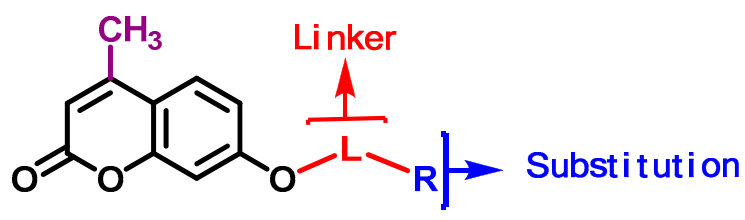

Figure 1. Umbelliferone and its potential modification sites.

\section{Results and Discussion}

The preparation of umbelliferone (1) and 7-(4-bromoalkoxy)-4-methyl benzopyran-2-one (2c-g) was performed as per our previous methods. The rest of the UDs were synthesized according to the following method by an alkylation reaction, Friedel-Crafts reaction, and Claisen rearrangement reaction (Schemes 1-6) [14-16].<smiles>CCOC(=O)CC(C)=O</smiles>

Scheme 1. Synthesis of compounds 1-2g.

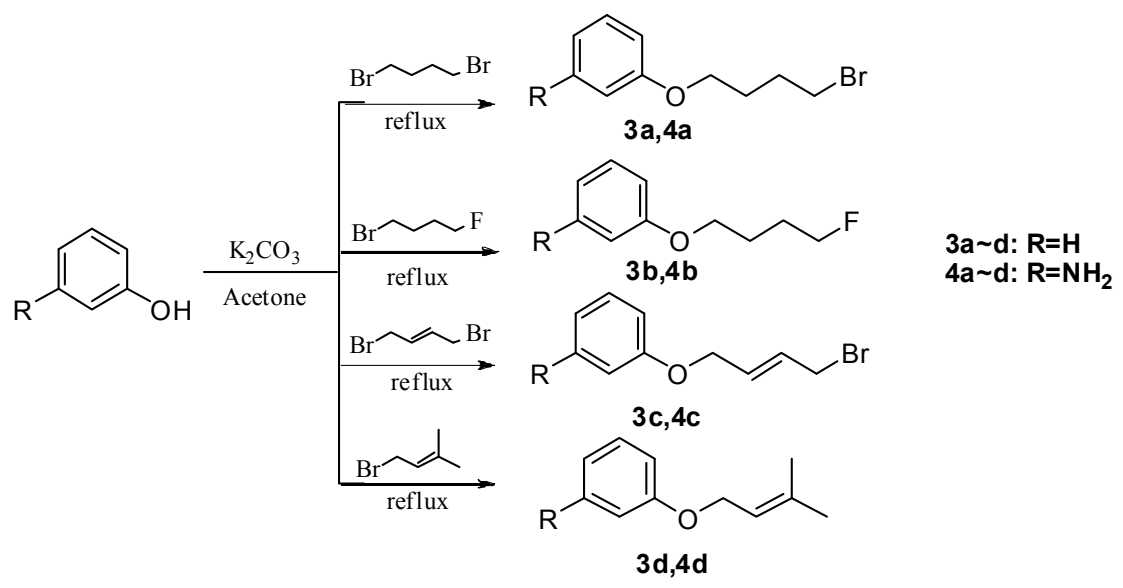

Scheme 2. Synthesis of compounds $3 a-4 d$. 
<smiles></smiles>

Scheme 3. Synthesis of compounds 5 and $\mathbf{6}$. Reagents and conditions: (a) $200{ }^{\circ} \mathrm{C}$; (b) 1,4-dibromobutane; $\mathrm{KOH}$; $\mathrm{KI}$ and TBAB; reflux in acetonitrile.<smiles>COC(=O)c1cccc(CCCCOc2ccc3c(C)cc(=O)oc3c2)c1</smiles>

Scheme 4. Synthesis of compound 7. Reagents and conditions: (a) $\mathrm{HCl}$, stirring at room temperature; (b) methanol; $\mathrm{H}_{2} \mathrm{SO}_{4}$; reflux; (c) compound 2c; $\mathrm{AlCl}_{3} ; 80{ }^{\circ} \mathrm{C}$.<smiles>Cc1cc(=O)oc2cc(OCCCCOc3cccc(N)c3)ccc12</smiles>

Scheme 5. Synthesis of compound 8. Reagents and conditions: $\mathrm{KOH}$; KI and TBAB; reflux in acetonitrile.

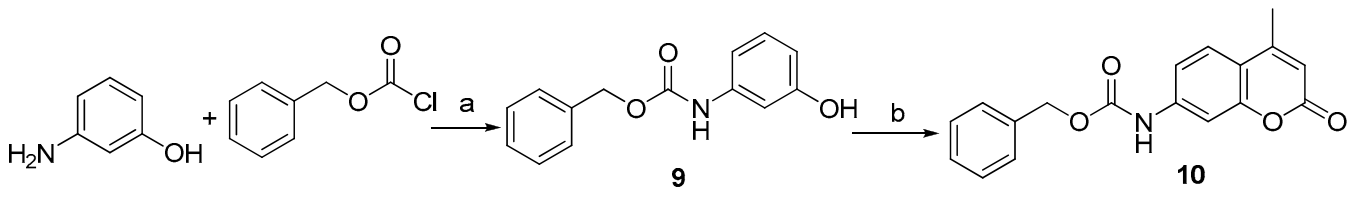

Scheme 6. Synthesis of compound 9 and 10. Reagents and conditions: (a) para-dioxane; stirred at $0{ }^{\circ} \mathrm{C}$; (b) ethyl acetoacetate; phosphoric acid; $80{ }^{\circ} \mathrm{C}$.

Fungicidal activity and structure-activity relationship: The fungicidal activity of the compounds is shown in Table 1. As we had predicted, 4-methylumbelliferone (1) and its 7-subsituted derivatives exhibited significant inhibitory effects at the concentration of $100 \mathrm{mg} / \mathrm{L}$. In particular, compound $\mathbf{2 f}$ exhibited a significant activity with inhibitory rate up to $90 \%$, and compounds $\mathbf{2} \mathbf{a}-\mathbf{e}$ and $\mathbf{2} \mathbf{g}$ displayed moderate to strong activity against all tested fungi. The $\mathrm{EC}_{50}$ values of derivatives were evaluated at a series of lower concentration, which are shown in Table 2. The results indicated that compound $\mathbf{2 f}$ had the lowest $\mathrm{EC}_{50}$ values of $50.5 \mu \mathrm{mol} / \mathrm{L}, 30.7 \mu \mathrm{mol} / \mathrm{L}$, and $58.9 \mu \mathrm{mol} / \mathrm{L}$ against A. alternata, B. cinerea, and F. oxysporum, respectively. 
Table 1. In vitro fungicidal activities of the prepared compounds at $100 \mathrm{ug} / \mathrm{mL}$ ( $96 \mathrm{~h}$ of incubation).

\begin{tabular}{cccc}
\hline \multirow{2}{*}{ Compd. } & \multicolumn{2}{c}{ The Inhibition Rate (\%; mean \pm SD; $\mathbf{N}=\mathbf{3})$} \\
\cline { 2 - 4 } & A. Alternata & B. Cinerea & F. Oxysporum \\
\hline $\mathbf{1}$ & $38.5 \pm 4.38$ & $25.8 \pm 3.8$ & $41.2 \pm 2.5$ \\
$\mathbf{2 a}$ & $38.1 \pm 5.9$ & $39.6 \pm 6.2$ & $33.2 \pm 4.4$ \\
$\mathbf{2 b}$ & $42.9 \pm 6.7$ & $36.3 \pm 5.8$ & $28.6 \pm 3.4$ \\
$\mathbf{2 c}$ & $70.6 \pm 5.5$ & $68.3 \pm 4.6$ & $60.4 \pm 5.3$ \\
$\mathbf{2 d}$ & $40.3 \pm 3.7$ & $46.2 \pm 5.8$ & $35.5 \pm 7.2$ \\
$\mathbf{2 e}$ & $63.7 \pm 3.9$ & $72.9 \pm 3.5$ & $62.8 \pm 5.6$ \\
$\mathbf{2 f}$ & $81.5 \pm 3.7$ & $90.99 \pm 2.3$ & $84.8 \pm 3.2$ \\
$\mathbf{2 g}$ & $48.4 \pm 4.0$ & $70.6 \pm 5.2$ & $53.5 \pm 4.2$ \\
$\mathbf{3 a}$ & $49.6 \pm 3.6$ & $41.2 \pm 5.4$ & $50.2 \pm 6.3$ \\
$\mathbf{3 b}$ & $3.8 \pm 2.8$ & $10.2 \pm 2.6$ & $8.4 \pm 4.1$ \\
$\mathbf{3 c}$ & $58.2 \pm 3.6$ & $63.0 \pm 7.0$ & $61.3 \pm 3.8$ \\
$\mathbf{3 d}$ & $29.6 \pm 5.3$ & $32.5 \pm 6.2$ & $26.2 \pm 4.9$ \\
$\mathbf{4 a}$ & $30.5 \pm 5.2$ & $32.1 \pm 4.5$ & $28.4 \pm 5.8$ \\
$\mathbf{4 b}$ & $35.3 \pm 5.6$ & $46.2 \pm 3.7$ & $33.2 \pm 3.1$ \\
$\mathbf{4 c}$ & $12.6 \pm 2.6$ & $15.0 \pm 6.4$ & $13.7 \pm 4.2$ \\
$\mathbf{4 d}$ & $10.2 \pm 3.5$ & $12.6 \pm 4.5$ & $9.8 \pm 2.6$ \\
$\mathbf{5}$ & $22.4 \pm 4.7$ & $26.4 \pm 2.1$ & $32.6 \pm 5.6$ \\
$\mathbf{6}$ & $27.6 \pm 8.3$ & $35.93 \pm 1.73$ & $29.8 \pm 3.8$ \\
$\mathbf{7}$ & $20.8 \pm 7.7$ & $21.9 \pm 3.0$ & $18.7 \pm 4.1$ \\
$\mathbf{8}$ & $26.6 \pm 3.9$ & $18.9 \pm 8.5$ & $25.3 \pm 5.2$ \\
$\mathbf{9}$ & $58.5 \pm 3.4$ & $70.9 \pm 1.7$ & $77.5 \pm 3.4$ \\
$\mathbf{1 0}$ & $77.3 \pm 5.5$ & $84.4 \pm 3.5$ & $80.5 \pm 5.7$ \\
DMSO (1\%) & 0 & 0 & 0 \\
carbendazim & $90.7 \pm 8.18$ & $94.3 \pm 6.4$ & $91.4 \pm 5.4$ \\
\hline & & &
\end{tabular}

Table 2. $\mathrm{EC}_{50}$ values and $\log \mathrm{P}$ of effective compounds against three phytopathogenic fungi.

\begin{tabular}{ccccc}
\hline \multirow{2}{*}{ Compound } & \multirow{2}{*}{$\mathbf{L o g} \mathbf{P}$} & \multicolumn{3}{c}{ EC $_{\mathbf{5 0}}(\mathbf{9 5} \% \mathbf{C I} \boldsymbol{\mu m o l} / \mathbf{L})$} \\
\cline { 3 - 5 } & & A. Alternata & B. Cinerea & $\boldsymbol{F}$. Oxysporum \\
\hline $\mathbf{1}$ & 1.61 & $922.4(827.6-1098.9)$ & $1037.1(955.3-1201.1)$ & $1165.9(1076.2-1268.1)$ \\
$\mathbf{2 c}$ & 3.26 & $193.5(145.6-258.7)$ & $221.1(137.2-270.9)$ & $245.2(180.9-298.2)$ \\
$\mathbf{2} \mathbf{e}$ & 2.62 & $381.6(282.1-466.7)$ & $360.4(274.1-462.7)$ & $405.9(311.3-505.9)$ \\
$\mathbf{2 f}$ & 3.22 & $50.5(28.5-82.2)$ & $30.7(15.5-47.5)$ & $58.9(30.4-89.3)$ \\
$\mathbf{2 g}$ & 3.11 & $232.1(158.8-336.9)$ & $280.8(206.7-350.4)$ & $207.1(149.8-279.2)$ \\
$\mathbf{9}$ & 2.87 & $185.8(117.6-254.0)$ & $130.7(76.0-195.7)$ & $182.9(145.9-248.2)$ \\
$\mathbf{1 0}$ & 3.23 & $105.1(69.5-176.2)$ & $66.6(50.7-115.1)$ & $60.8(34.6-120.6)$ \\
Carbendazim & 1.29 & $18.8(6.8-30.0)$ & $20.6(7.8-32.4)$ & $33.5(14.6-49.7)$ \\
\hline
\end{tabular}

It is worth noting that the side chain was important to the activity exhibition. As the length of the side chain increased from two to five carbon atoms, the activity varied with nonlinear changes. It was clearly seen that four carbon atoms was optimum on activity exhibition, which was in accordance with our previous study. In order to further confirm the contribution of the coumarin skeleton, its core structure of benzopyran-2-one was substituted by benzene (3a-d) and aniline (4a-d). The activity was found to be significantly decreased compared with the related umbelliferone derivatives, except (4-bromobutoxy) benzene (3a). Therefore, the results proved that taking umbelliferone as the main skeleton, together with our proposed modification strategy, was evidently effective.

Based on the above findings, a series of coumarin derivatives were designed and screened for their antifungal activity. The results indicated that the functional groups played an important role in activity exhibition. Halides, especially fluoro compounds, are widely applied in lead compound modification and have been proven to show good biocompatibility and activity [17]. In this study, it was found that the inhibition rate increased significantly with compound $2 \mathrm{e}$ (about $65 \%$ ) compared to compound 1 (about 30\%) when the terminal bromine was substituted with fluorine. We also deemed it meaningful to 
introduce different fluoro moieties in our studies. Isopentenyl has been reported to play an important role in membrane binding and activity expression of biomolecules $[18,19]$. Our results showed similar results, with activity being enhanced when isopentenyl was introduced as a terminal moiety. Methoxyacrylate, originated from strobilurin A, is a key skeleton in commercial fungicides [20,21]. By coupling methoxyacrylate with coumarin with four carbon chain (7), we managed to achieve good activity. The type of linkage also played an important role in antifungi activity. The results indicated that the inhibitory effect of compound $\mathbf{2} \mathbf{f}$ was much better than compound $\mathbf{2} \mathbf{c}$ due to the slight structural difference between the two, with compound $\mathbf{2} \mathbf{f}$ having an olefinic linkage instead of the alkoxy side chain of compound $2 \mathrm{c}$. We propose that the increase in activity may have been induced by the conjugation of double bond, $\mathrm{O}$ atom, and aromatic ring in compound $\mathbf{2 f}$.

\section{Experimental Section}

All starting chemicals were purchased from commercial vendors, were of AR grade, and were used without purification. Reactions were monitored by thin-layer chromatography (Silica Gel 60 F254). Target compounds were purified with column chromatography using silica gel (200-300 mesh). ${ }^{1} \mathrm{H}-\mathrm{NMR}(400 \mathrm{MHz})$ and ${ }^{13} \mathrm{C}-\mathrm{NMR}(100 \mathrm{MHz})$ spectra were recorded on a Bruker AM-400BB spectrometer (Bruker, Karlsruhe, Germany) in $\mathrm{CDCl}_{3} / \mathrm{DMSO}-d_{6}$ solution with TMS as the internal standard. The chemical shift values $(\delta)$ below are listed in ppm and the coupling constant values $(J)$ are in Hertz. The melting points were determined with a X-4 melting point apparatus. The purity of all test compounds was above $95 \%$. Compounds $\mathbf{2 a}$ and $\mathbf{2} \mathbf{b}$ were prepared as per our previous study and used directly [13].

\subsection{General Procedure for the Preparation of Compounds $\mathbf{2} \mathbf{c}-\mathbf{g}$}

1,4-Dibromobutane, 1,5-dibromopentane, 1-bromo-4-fluorobutane, (2E)-1,4-dibromo-2-butene (3.0 mmol), or 1-bromo-3-methyl-2-butene $(5.0 \mathrm{mmol})$ was added to a solution of 4-methylcoumarin $(3.5 \mathrm{mmol}), \mathrm{KOH}(1 \mathrm{mmol})$, and $\mathrm{K}_{2} \mathrm{CO}_{3}(4 \mathrm{mmol})$ in acetone $(20 \mathrm{~mL})$, respectively. The reaction was heated at reflux detected with TLC for $5 \mathrm{~h}$. Then, the crude products were prepared by filtering the mixture and evaporating the solvent and were further purified by silica chromatography $\left(\mathrm{CHCl}_{3} / \mathrm{MeOH} 50 / 1\right)$ to give compounds $\mathbf{2} \mathbf{c}-\mathbf{g}$ (Scheme 1$)$.

7-(4-Bromobutoxy)-4-methylbenzopyran-2-one (2c): White solid; yield: 65\%; m.p., $42-44{ }^{\circ} \mathrm{C}$; ESI-MS $\mathrm{m} / \mathrm{z}$ : $312.2[\mathrm{M}+\mathrm{H}]^{+} .{ }^{1} \mathrm{H}-\mathrm{NMR}\left(\mathrm{CHCl}_{3}-\mathrm{d}, 400 \mathrm{MHz}\right) \delta: 1.98-2.02(\mathrm{~m}, 2 \mathrm{H}), 2.06-2.12(\mathrm{~m}, 2 \mathrm{H}), 2.40(\mathrm{~s}, 3 \mathrm{H})$, $3.50(\mathrm{t}, 2 \mathrm{H}, J=8.0 \mathrm{~Hz}), 4.06(\mathrm{t}, 2 \mathrm{H}, J=8.0 \mathrm{~Hz}), 6.14(\mathrm{~s}, 1 \mathrm{H}), 6.80(\mathrm{~d}, 1 \mathrm{H}, J=4.0 \mathrm{~Hz}), 6.82(\mathrm{dd}, 1 \mathrm{H}$, $J=8.0 \mathrm{~Hz}), 7.49(\mathrm{~d}, 1 \mathrm{H}, J=8.0 \mathrm{~Hz}) ;{ }^{13} \mathrm{C}-\mathrm{NMR}\left(\mathrm{CHCl}_{3}-\mathrm{d}, 100 \mathrm{MHz}\right) \delta: 27.69,29.34,32.16,67.56,101.41$, $112.61,112.87,113.18,128.79,143.36,155.93,161.15,162.09$.

7-(4-Bromopentyloxy)-4-methylbenzopyran-2-one (2d): White solid; yield: 60\%; m.p., 60-62 ${ }^{\circ} \mathrm{C}$; ESI-MS $m / z: 326.2[\mathrm{M}+\mathrm{H}]^{+} .{ }^{1} \mathrm{H}-\mathrm{NMR}\left(\mathrm{CHCl}_{3}-\mathrm{d}, 400 \mathrm{MHz}\right) \delta: 1.30-1.34(\mathrm{~m}, 2 \mathrm{H}), 1.92-1.98(\mathrm{~m}, 2 \mathrm{H}), 2.02-2.06$ $(\mathrm{m}, 2 \mathrm{H}), 2.42(\mathrm{~s}, 3 \mathrm{H}), 3.51(\mathrm{t}, 2 \mathrm{H}, J=8.0 \mathrm{~Hz}), 4.08(\mathrm{t}, 2 \mathrm{H}, J=8.0 \mathrm{~Hz}), 6.15(\mathrm{~s}, 1 \mathrm{H}), 6.82(\mathrm{~d}, 1 \mathrm{H}, J=2.4 \mathrm{~Hz})$, $6.88(\mathrm{dd}, 1 \mathrm{H}, J=8.4 \mathrm{~Hz}, 2.4 \mathrm{~Hz}), 7.50(\mathrm{~d}, 1 \mathrm{H}, J=8.4 \mathrm{~Hz})$.

7-(4-Fluorobutoxy)-4-methylbenzopyran-2-one (2e): White solid; yield: 63\%; m.p., $116-118{ }^{\circ} \mathrm{C}$; ESI-MS $\mathrm{m} / z: 251.3[\mathrm{M}+\mathrm{H}]^{+} .{ }^{1} \mathrm{H}-\mathrm{NMR}\left(\mathrm{CHCl}_{3}-\mathrm{d}, 400 \mathrm{MHz}\right) \delta: 1.86-1.98(\mathrm{~m}, 4 \mathrm{H}), 2.40(\mathrm{~s}, 3 \mathrm{H}), 4.07(\mathrm{t}, 2 \mathrm{H}$, $J=8.0 \mathrm{~Hz}), 4.79(\mathrm{t}, 2 \mathrm{H}, J=8.0 \mathrm{~Hz}), 6.13(\mathrm{~s}, 1 \mathrm{H}), 6.80(\mathrm{~d}, 1 \mathrm{H}, J=2.4 \mathrm{~Hz}), 6.85(\mathrm{dd}, 1 \mathrm{H}, J=8.8 \mathrm{~Hz}, 2.4 \mathrm{~Hz})$, $7.49(\mathrm{~d}, 1 \mathrm{H}, J=8.8 \mathrm{~Hz})$.

7-(4-Bromobutenyloxy)-4-methylbenzopyran-2-one (2f): White solid; yield: 65\%; m.p., $116-118{ }^{\circ} \mathrm{C}$ ESI-MS $m / z: 310.2[\mathrm{M}+\mathrm{H}]^{+} .{ }^{1} \mathrm{H}-\mathrm{NMR}\left(\mathrm{CHCl}_{3}-\mathrm{d}, 400 \mathrm{MHz}\right) \delta: 2.40(\mathrm{~s}, 3 \mathrm{H}), 4.00(\mathrm{~d}, 2 \mathrm{H}, J=7.2 \mathrm{~Hz}), 4.62(\mathrm{~d}, 2 \mathrm{H}$, $J=4.8 \mathrm{~Hz}), 6.01-6.15(\mathrm{~m}, 2 \mathrm{H}), 6.15(\mathrm{~s}, 1 \mathrm{H}), 6.80(\mathrm{~d}, 1 \mathrm{H}, J=2.0 \mathrm{~Hz}), 6.87(\mathrm{dd}, 1 \mathrm{H}, J=8.8 \mathrm{~Hz}, 2.4 \mathrm{~Hz})$, $7.55(\mathrm{~d}, 1 \mathrm{H}, J=8.8 \mathrm{~Hz})$. 
7-(4-Isopentenyloxy)-4-methylbenzopyran-2-one (2g): White solid; yield: 75\%; ESI-MS m/z: $247.3[\mathrm{M}+\mathrm{H}]^{+}$. ${ }^{1} \mathrm{H}-\mathrm{NMR}\left(\mathrm{CHCl}_{3}-\mathrm{d}, 400 \mathrm{MHz}\right) \delta: 1.77(\mathrm{~s}, 3 \mathrm{H}), 1.81(\mathrm{~s}, 3 \mathrm{H}), 2.49(\mathrm{~s}, 3 \mathrm{H}), 4.58(\mathrm{~d}, 2 \mathrm{H}, J=8.0 \mathrm{~Hz}), 5.47-5.50$ $(\mathrm{m}, 1 \mathrm{H}), 6.13(\mathrm{~s}, 1 \mathrm{H}), 6.82(\mathrm{~d}, 1 \mathrm{H}, J=2.0 \mathrm{~Hz}), 6.87(\mathrm{dd}, 1 \mathrm{H}, J=8.8 \mathrm{~Hz}, J=2.4 \mathrm{~Hz}), 7.49(\mathrm{~d}, 1 \mathrm{H}, J=8.8 \mathrm{~Hz})$.

\subsection{General Procedure for the Preparation of Compounds $\mathbf{3 a - d}$ and $\mathbf{4 a - d}$}

1,4-Dibromobutane, 1-bromo-4-fluorobutane, (2E)-1,4-dibromo-2-butene or 1-bromo-3-methyl2-butene( $3.0 \mathrm{mmol})$ was added to a solution of benzene (3a-d) or aniline (4a-d) $(3.5 \mathrm{mmol}), \mathrm{KOH}$ $(1 \mathrm{mmol})$, and $\mathrm{K}_{2} \mathrm{CO}_{3}(4 \mathrm{mmol})$ in acetone $(20 \mathrm{~mL})$, respectively. The reaction was heated at reflux detected with TLC. Then, the crude products were further purified by silica chromatography $\left(\mathrm{CHCl}_{3} / \mathrm{MeOH} 50 / 1\right)$ to give compounds $3 \mathbf{a}-\mathbf{d}$ and $\mathbf{4 a}-\mathbf{d}$ (Scheme 2).

(4-Bromobutoxy) benzene (3a): White oil; yield: 75\%; m.p., 40-41 ${ }^{\circ} \mathrm{C} ;{ }^{1} \mathrm{H}-\mathrm{NMR}\left(\mathrm{CHCl}_{3}-\mathrm{d}, 400 \mathrm{MHz}\right)$ $\delta: 1.74-1.78(\mathrm{~m}, 2 \mathrm{H}), 1.80-1.86(\mathrm{~m}, 2 \mathrm{H}), 3.51(\mathrm{t}, 2 \mathrm{H}, J=8.0 \mathrm{~Hz}), 4.08(\mathrm{t}, 2 \mathrm{H}, J=8.0 \mathrm{~Hz}), 6.99(\mathrm{dd}, 2 \mathrm{H}$, mboxemphJ $=8.0 \mathrm{~Hz}, 2.4 \mathrm{~Hz}), 7.03(\mathrm{dd}, 1 \mathrm{H}, J=8.0 \mathrm{~Hz}, 2.4 \mathrm{~Hz}), 7.37-7.40(\mathrm{~m}, 2 \mathrm{H})$.

(4-Fluorobutoxy) benzene (3b): White oil; yield: $65 \%$; m.p., $55-57{ }^{\circ} \mathrm{C} ;{ }^{1} \mathrm{H}-\mathrm{NMR}\left(\mathrm{CHCl}_{3}-\mathrm{d}, 400 \mathrm{MHz}\right) \delta$ : $1.70-1.86(\mathrm{~m}, 4 \mathrm{H}), 4.07(\mathrm{t}, 2 \mathrm{H}, J=8.0 \mathrm{~Hz}), 4.50(\mathrm{t}, 2 \mathrm{H}, J=8.0 \mathrm{~Hz}), 6.96(\mathrm{dd}, 2 \mathrm{H}, J=8.0 \mathrm{~Hz}, 2.4 \mathrm{~Hz})$, $7.02(\mathrm{dd}, 1 \mathrm{H}, J=8.0 \mathrm{~Hz}, 2.4 \mathrm{~Hz}), 7.33-7.36(\mathrm{~m}, 2 \mathrm{H})$.

7-(4-Bromobutenyloxy) benzene (3c): White solid; yield: 72\%; m.p., $46-48{ }^{\circ} \mathrm{C} ;{ }^{1} \mathrm{H}-\mathrm{NMR}\left(\mathrm{CHCl}_{3}\right.$-d, $400 \mathrm{MHz}) \delta: 4.02(\mathrm{~d}, 2 \mathrm{H}, J=7.2 \mathrm{~Hz}), 4.65(\mathrm{~d}, 2 \mathrm{H}, J=4.8 \mathrm{~Hz}), 6.00-6.12(\mathrm{~m}, 2 \mathrm{H}), 7.03(\mathrm{dd}, 1 \mathrm{H}, J=8.8 \mathrm{~Hz}$, $J=2.0 \mathrm{~Hz}), 7.05(\mathrm{dd}, 2 \mathrm{H}, J=8.8 \mathrm{~Hz}, J=2.0 \mathrm{~Hz}), 7.34-7.38(\mathrm{~m}, 1 \mathrm{H})$.

(4-Isopentenyloxy) benzene (3d): Yellow oil; yield: $76 \% ;{ }^{1} \mathrm{H}-\mathrm{NMR}\left(\mathrm{CHCl}_{3}-\mathrm{d}, 400 \mathrm{MHz}\right) \delta: 1.75(\mathrm{~s}, 3 \mathrm{H})$, $1.80(\mathrm{~s}, 3 \mathrm{H}), 4.59(\mathrm{~d}, 1 \mathrm{H}, J=8.0 \mathrm{~Hz}), 5.46(\mathrm{~m}, 1 \mathrm{H}), 7.02(\mathrm{dd}, 1 \mathrm{H}, J=8.8 \mathrm{~Hz}, J=2.4 \mathrm{~Hz}), 7.04(\mathrm{dd}, 2 \mathrm{H}$, $J=8.8 \mathrm{~Hz}, J=2.4 \mathrm{~Hz}), 7.34-7.37(\mathrm{~m}, 2 \mathrm{H})$.

(4-Bromobutoxy) aniline (4a): White solid; yield: 63\%; m.p., $116-118{ }^{\circ} \mathrm{C} ;{ }^{1} \mathrm{H}-\mathrm{NMR}\left(\mathrm{CHCl}_{3}-\mathrm{d}, 400 \mathrm{MHz}\right)$ $\delta: 1.74-1.82(\mathrm{~m}, 4 \mathrm{H}), 3.52(\mathrm{t}, 2 \mathrm{H}, J=8.0 \mathrm{~Hz}), 3.68(\mathrm{~s}, 2 \mathrm{H}), 4.09(\mathrm{t}, 2 \mathrm{H}, J=8.0 \mathrm{~Hz}), 6.16-6.19(\mathrm{~m}, 2 \mathrm{H})$, $6.35(\mathrm{dd}, 1 \mathrm{H}, J=8.0 \mathrm{~Hz}, 2.4 \mathrm{~Hz}), 7.09(\mathrm{dd}, 1 \mathrm{H}, J=8.4 \mathrm{~Hz}, 8.2 \mathrm{~Hz})$.

(4-Fluorobutoxy) aniline (4b): Yellow solid; yield: $60 \%$; m.p., $76-78{ }^{\circ} \mathrm{C} ;{ }^{1} \mathrm{H}-\mathrm{NMR}\left(\mathrm{CHCl}_{3}-\mathrm{d}, 400 \mathrm{MHz}\right) \delta$ : $1.62-1.78(\mathrm{~m}, 4 \mathrm{H}), 3.66(\mathrm{~s}, 2 \mathrm{H}), 4.06(\mathrm{t}, 2 \mathrm{H}, J=8.0 \mathrm{~Hz}), 4.52(\mathrm{t}, 2 \mathrm{H}, J=8.0 \mathrm{~Hz}), 6.21(\mathrm{dd}, 1 \mathrm{H}, J=8.0 \mathrm{~Hz}$, $2.4 \mathrm{~Hz}), 6.25(\mathrm{dd}, 1 \mathrm{H}, J=2.4 \mathrm{~Hz}, 2.0 \mathrm{~Hz}), 6.28-6.31(\mathrm{~m}, 1 \mathrm{H}), 7.02(\mathrm{dd}, 1 \mathrm{H}, J=8.4 \mathrm{~Hz}, 8.0 \mathrm{~Hz})$.

(4-Bromobutenyloxy) aniline (4c): Yellow solid; m.p., $121-123{ }^{\circ} \mathrm{C} ;{ }^{1} \mathrm{H}-\mathrm{NMR}\left(\mathrm{CHCl}_{3}-\mathrm{d}, 400 \mathrm{MHz}\right) \delta: 3.62$ (s, $2 \mathrm{H}), 4.02(\mathrm{~d}, 2 \mathrm{H}, J=7.2 \mathrm{~Hz}), 4.68(\mathrm{~d}, 2 \mathrm{H}, J=4.8 \mathrm{~Hz}), 6.00-6.12(\mathrm{~m}, 2 \mathrm{H}), 6.16-6.20(\mathrm{~m}, 1 \mathrm{H}), 6.22(\mathrm{dd}, 1 \mathrm{H}$, $J=2.4 \mathrm{~Hz}, 2.0 \mathrm{~Hz}), 6.38(\mathrm{~m}, 1 \mathrm{H}), 7.05(\mathrm{dd}, 1 \mathrm{H}, J=8.4 \mathrm{~Hz}, 8.0 \mathrm{~Hz})$.

(4-Isopentenyloxy) aniline (4d): Yellow solid; yield: $68 \%$; m.p.,68-70 ${ }^{\circ} \mathrm{C} ;{ }^{1} \mathrm{H}-\mathrm{NMR}\left(\mathrm{CHCl}_{3}-\mathrm{d}, 400 \mathrm{MHz}\right) \delta$ : $1.72(\mathrm{~s}, 3 \mathrm{H}), 1.84(\mathrm{~s}, 3 \mathrm{H}), 3.70(\mathrm{~s}, 2 \mathrm{H}), 4.56(\mathrm{~d}, 1 \mathrm{H}, J=8.0 \mathrm{~Hz}), 5.44(\mathrm{~m}, 1 \mathrm{H}), 6.14-6.21(\mathrm{~m}, 1 \mathrm{H}), 6.23(\mathrm{dd}$, $1 \mathrm{H}, J=8.4 \mathrm{~Hz}, 2.0 \mathrm{~Hz}), 6.35-6.41(\mathrm{~m}, 1 \mathrm{H}), 7.07(\mathrm{dd}, 1 \mathrm{H}, J=8.4 \mathrm{~Hz}, 8.0 \mathrm{~Hz})$.

\subsection{General Procedure for the Preparation of Compounds $\mathbf{5}$ and $\mathbf{6}$}

Compound $2 \mathrm{~g}$ was added into single-necked flask and heated to $200{ }^{\circ} \mathrm{C}$. Then, the mixture was dissolved into methanol and purified by silica chromatography to get compound $\mathbf{5}$. Then, compound 5 $(1.5 \mathrm{mmol})$ was added to a solution of 1, 4-dibromobutane $(2 \mathrm{mmol}), \mathrm{KOH}(1 \mathrm{mmol})$, and $\mathrm{K}_{2} \mathrm{CO}_{3}$ $(2 \mathrm{mmol})$ in acetone $(10 \mathrm{~mL})$. The reaction was heated at reflux for $3 \mathrm{~h}$. The crude products were prepared by filtering the mixture and evaporating the solvent and were further purified by silica chromatography to give compound 6 . 
7-Hydroxy-6-isopentenyl-4-methylbenzopyran-2-one (5): White solid; yield: 30\%; m.p., 83-84 ${ }^{\circ} \mathrm{C}$; ESI-MS $m / z: 245.3\left[\mathrm{M}+\mathrm{H}^{+} .{ }^{1} \mathrm{H}-\mathrm{NMR}\left(\mathrm{CHCl}_{3}-\mathrm{d}, 400 \mathrm{MHz}\right) \delta: 1.70(\mathrm{~s}, 3 \mathrm{H}), 1.83(\mathrm{~s}, 3 \mathrm{H}), 2.41(\mathrm{~s}, 3 \mathrm{H}), 3.30(\mathrm{~d}, 2 \mathrm{H}\right.$, $J=6.0 \mathrm{~Hz}), 4.08(\mathrm{t}, 1 \mathrm{H}, J=6.0 \mathrm{~Hz}), 6.09(\mathrm{~s}, 1 \mathrm{H}), 6.69(\mathrm{~d}, 1 \mathrm{H}, J=2.0 \mathrm{~Hz}), 6.79-6.82(\mathrm{dd}, 1 \mathrm{H}, J=8.8 \mathrm{~Hz}$, $J=2.4 \mathrm{~Hz}), 7.58(\mathrm{~d}, 1 \mathrm{H}, J=8.8 \mathrm{~Hz}) ;{ }^{13} \mathrm{C}-\mathrm{NMR}\left(\mathrm{CHCl}_{3}-\mathrm{d}, 100 \mathrm{MHz}\right) \delta: 17.24,19.34,22.4,24.62,102.06$, $109.82,112.44,112.94,115.1,125.20,126.03154 .56,155.15,161.63,162.46$.

1,4-Di(6-isopentenyl-4-methylbenzopyran-2-one-7-oxy)butane (6): White solid; yield: 20\%; m.p.,162-164 ${ }^{\circ} \mathrm{C}$; ESI-MS m/z: $529.6[\mathrm{M}+\mathrm{H}]^{+} .{ }^{1} \mathrm{H}-\mathrm{NMR}\left(\mathrm{CHCl}_{3}-\mathrm{d}, 400 \mathrm{MHz}\right) \delta: 1.28(\mathrm{~s}, 6 \mathrm{H}), 1.35(\mathrm{~s}, 6 \mathrm{H}), 2.03-2.07(\mathrm{~m}$, $4 \mathrm{H}), 2.44(\mathrm{~s}, 6 \mathrm{H}, J=6.0 \mathrm{~Hz}), 3.26(\mathrm{~d}, 4 \mathrm{H}, J=6.0 \mathrm{~Hz}), 4.05(\mathrm{~d}, 2 \mathrm{H}, J=8.0 \mathrm{~Hz}), 4.60(\mathrm{~d}, 2 \mathrm{H}, J=8.0 \mathrm{~Hz})$, $6.06(\mathrm{~s}, 2 \mathrm{H}), 6.16(\mathrm{~s}, 2 \mathrm{H}), 6.90(\mathrm{~d}, 2 \mathrm{H}, J=8.8 \mathrm{~Hz}), 6.98(\mathrm{dd}, 2 \mathrm{H}, J=8.8 \mathrm{~Hz}, J=2.0 \mathrm{~Hz}), 7.68(\mathrm{~d}, 2 \mathrm{H}$, $J=9.6 \mathrm{~Hz}) ;{ }^{13} \mathrm{C}-\mathrm{NMR}\left(\mathrm{CHCl}_{3}-\mathrm{d}, 100 \mathrm{MHz}\right) \delta: 17.25,19.11,19.33,24.92,30.68,63.69,67.78,101.25,112.69$, $125.95,127.62,128.17,128.98,129.84,154.29,155.00,162.11$

\subsection{General Procedure for the Preparation of Compound 7}

Benzoylcyanide ( $3 \mathrm{mmol}$ ) was dissolved in hydrochloric acid $(10 \mathrm{~mL})$ with stirring at room temperature for $2 \mathrm{~h}$. Then, the products were filtered out and added into methanol. Concentrated $\mathrm{H}_{2} \mathrm{SO}_{4}(1 \mathrm{~mL})$ was added to the mixture and refluxed. After that, the mixture was extracted with ethyl acetate and evaporated to yield the crude products, which reacted with compound 2c without purification catalyzed by $\mathrm{AlCl}_{3}$. The crude products were further purified by means of silica chromatography to provide compound 7 (Scheme 2).

1-(4-Methylbenzopyran-2-one-7-oxy)-4-(3-methylphenylglyoxylate)butane (7): White solid; yield: 30\%; m.p.,117-119 ${ }^{\circ} \mathrm{C}$; ESI-MS m/z: $395.4[\mathrm{M}+\mathrm{H}]^{+} .{ }^{1} \mathrm{H}-\mathrm{NMR}\left(\mathrm{CHCl}_{3}-\mathrm{d}, 400 \mathrm{MHz}\right) \delta: 1.22-1.27(\mathrm{~m}, 2 \mathrm{H})$, 1.71-1.79 (m, 2H), $2.40(\mathrm{~s}, 3 \mathrm{H}), 2.86-2.91(\mathrm{~m}, 2 \mathrm{H}), 3.68(\mathrm{~s}, 3 \mathrm{H}), 4.14-4.18(\mathrm{~m}, 2 \mathrm{H}), 6.16(\mathrm{~s}, 1 \mathrm{H}), 6.85(\mathrm{dd}$, $1 \mathrm{H}, J=8.4 \mathrm{~Hz}, 2.4 \mathrm{~Hz}), 7.48(\mathrm{t}, 1 \mathrm{H}, J=8.4 \mathrm{~Hz}), 7.57(\mathrm{dd}, 1 \mathrm{H}, J=8.0 \mathrm{~Hz}, 2.4 \mathrm{~Hz}), 7.62(\mathrm{t}, 1 \mathrm{H}, J=8.4 \mathrm{~Hz})$, 7.70-7.73 (m, 2H), 8.11(d, 1H, $J=8.0 \mathrm{~Hz}) ;{ }^{13} \mathrm{C}-\mathrm{NMR}\left(\mathrm{CHCl}_{3}-\mathrm{d}, 100 \mathrm{MHz}\right) \delta: 18.73,22.73,29.71,31.68$, $52.34,68.70,103.39,111.63,112.50,113.07,113.57,125.41,125.96,128.51,130.20,133.72,145.2,152.7$, $153.15,155.12,159.71,161.89,170.43$.

\subsection{General Procedure for the Preparation of Compound $\mathbf{8}$}

Compound $2 \mathrm{c}(2.0 \mathrm{mmol})$ was added to a solution of 3-aminophenol $(2.5 \mathrm{mmol}), \mathrm{KOH}(1 \mathrm{mmol})$, and $\mathrm{K}_{2} \mathrm{CO}_{3}(3 \mathrm{mmol})$ in acetone $(10 \mathrm{~mL})$. The mixture was heated at reflux for $3 \mathrm{~h}$ and the reaction was detected with TLC. The mixture was extracted with ethyl acetate and evaporated to get crude product. The crude products were prepared by filtering the mixture and evaporating the solvent and were further purified by silica chromatography to give compound 8 (Scheme 3).

1-(4-Methylbenzopyran-2-one-7-oxy)-4-(3-aminophenol) butane (8): White solid; yield: 54\%; m.p., 123-125 ${ }^{\circ} \mathrm{C}$; ESI-MS m/z: $340.4[\mathrm{M}+\mathrm{H}]^{+} .{ }^{1} \mathrm{H}-\mathrm{NMR}\left(\mathrm{CHCl}_{3}-\mathrm{d}, 400 \mathrm{MHz}\right) \delta: 1.23-1.28(\mathrm{~m}, 2 \mathrm{H}), 2.02-2.10$ $(\mathrm{m}, 2 \mathrm{H}), 2.40(\mathrm{~s}, 3 \mathrm{H}), 4.11(\mathrm{~s}, 2 \mathrm{H}), 4.51-4.57(\mathrm{~m}, 2 \mathrm{H}), 4.62-4.66(\mathrm{~m}, 2 \mathrm{H}), 6.02-6.09(\mathrm{~m}, 1 \mathrm{H}), 6.14(\mathrm{~s}, 1 \mathrm{H})$, 6.19-6.34 (m, 2H), 6.85-6.89 (m, 1H), $7.05(\mathrm{t}, 1 \mathrm{H}, J=8.4 \mathrm{~Hz}), 7.48(\mathrm{t}, 2 \mathrm{H}, J=8.8 \mathrm{~Hz}) ;{ }^{13} \mathrm{C}-\mathrm{NMR}\left(\mathrm{CHCl}_{3}-\mathrm{d}\right.$, $100 \mathrm{MHz}) \delta:$ 18.69, 27.8(2), 67.32(2), 101.74, 104.69, 108.29, 112.08, 113.73, 126.88, 128.43, 129.56, 130.16, $147.81,152.59,155.12,159.71,161.34,161.50$.

\subsection{General Procedure for the Preparation of Compounds $\mathbf{9}$ and $\mathbf{1 0}$}

The amine $(5.0 \mathrm{mmol})$ was dissolved in water, and $\mathrm{NaHCO}_{3}(10.0 \mathrm{mmol})$ was added with stirring. After being cooled to $5^{\circ} \mathrm{C}, \mathrm{Cbz}-\mathrm{Cl}(7.5 \mathrm{mmol})$ was added slowly in para-dioxane. Then, the mixture was stirred at $0{ }^{\circ} \mathrm{C}$ for $1 \mathrm{~h}$ and allowed to warm to room temperature for $12 \mathrm{~h}$. After the reaction, water was added and extracted with ethyl acetate. The aqueous layers were acidified to $\mathrm{pH}$ of 1 and extracted three times with ethyl acetate. The organic layers were dried with sodium sulfate, and the solvent was evaporated to get compound 9 . Ethyl acetoacetate $(2 \mathrm{mmol})$ was added to a mixture of compound $9(1 \mathrm{mmol})$ and phosphoric acid $(10 \mathrm{~mL})$. The mixture was heated with stirring at $80{ }^{\circ} \mathrm{C}$ for 
$5 \mathrm{~h}$. After cooling down, the mixture was quenched with ice-water. The crude product was filtered out, dried, and purified by recrystallization from ethanol to yield compound $\mathbf{1 0}$ (Scheme 4).

N-Carbobenzoxy-3-hydroxyaniline (9): White solid; yield: 68\%; ESI-MS m/z: $244.3[\mathrm{M}+\mathrm{H}]^{+}$. m.p., 204-206 ${ }^{\circ} \mathrm{C} ;{ }^{1} \mathrm{H}-\mathrm{NMR}\left(\mathrm{CHCl}_{3}-\mathrm{d}, 400 \mathrm{MHz}\right) \delta: 4.62(\mathrm{~s}, 2 \mathrm{H}), 5.35(\mathrm{~s}, 1 \mathrm{H}), 6.65(\mathrm{dd}, 1 \mathrm{H}, J=8.0, \mathrm{~Hz}, 2.0 \mathrm{~Hz})$, $7.12(\mathrm{dd}, 1 \mathrm{H}, J=8.0 \mathrm{~Hz}, 2.0 \mathrm{~Hz}), 7.25-7.38(\mathrm{~m}, 5 \mathrm{H}), 7.47(\mathrm{dd}, 2 \mathrm{H}, J=8.0 \mathrm{~Hz}, 2.0 \mathrm{~Hz}), 9.10(\mathrm{~s}, 1 \mathrm{H})$; ${ }^{13} \mathrm{C}-\mathrm{NMR}\left(\mathrm{CHCl}_{3}-\mathrm{d}, 100 \mathrm{MHz}\right)$ 8: 68.76, 105.10, 113.42, 114.18, 127.26(2), 127.60, 128.92(2), 130.20, $136.15,137.30,153.38,158.78$.

4-Methyl-7-(N-carbobenzoxy)-aminocoumarin (10): White solid; yield: 28\%; m.p., 228-230 ${ }^{\circ} \mathrm{C}$; ESI-MS $\mathrm{m} / z: 310.4[\mathrm{M}+\mathrm{H}]^{+} .{ }^{1} \mathrm{H}-\mathrm{NMR}\left(\mathrm{CHCl}_{3}-\mathrm{d}, 400 \mathrm{MHz}\right) \delta: 2.40(\mathrm{~s}, 3 \mathrm{H}), 5.23(\mathrm{~s}, 2 \mathrm{H}), 6.19(\mathrm{~s}, 1 \mathrm{H}), 6.97(\mathrm{~s}$, $1 \mathrm{H}), 7.37-7.42(\mathrm{~m}, 5 \mathrm{H}), 7.44(\mathrm{~d}, 1 \mathrm{H}, J=1.6 \mathrm{~Hz}), 7.52(\mathrm{~d}, 2 \mathrm{H}, J=8.4 \mathrm{~Hz}) ;{ }^{13} \mathrm{C}-\mathrm{NMR}\left(\mathrm{CHCl}_{3}-\mathrm{d}, 100 \mathrm{MHz}\right)$ $\delta: 18.69,67.62,106.96,113.24,114.36,115.60,125.39,128.45(2), 128.59,128.72$ (2), 135.57, 141.32, 152.19, $152.77,154.58,161.06$.

\subsection{Antifungal Activity}

The fungicidal activities were investigated with three plant parasitic fungi (Alternaria alternata, Botrytis cinerea, and Fusarium oxysporum), and mycelial inhibition of radial growth on PDA media was employed [22,23].

\section{Conclusions}

In summary, the umbelliferone skeleton was revealed to play a key role in the antifungal activity. The structure-activity relationship study showed that coumarin coupled with haloalkane, methoxyacrylate, and isopentenyl moieties could significantly enhance activity. The research provides a good guidance for further study of the molecular design and discovery of allelochemical-based agents for phytopathogenic fungi control.

Author Contributions: Conceptualization, L.P.; Investigation, D.L., Q.Y. and Y.H.; Writing-Original Draft Preparation, L.P.; Writing-Review \& Editing, L.P.; Supervision, L.P. and L.J.

Funding: This work was supported by the Tianchi Talent on Doctor scheme introduced in the Xinjiang Uygur Autonomous Region of China, Research Foundation for Advanced Talents in Xinjiang Uygur Autonomous Region of China, and the Prophase-Sustentation Foundation of Xinjiang Agricultural University (No. XJAU201606).

Acknowledgments: The authors wish to thank Xiuzhuang Li and Hui Jin (Lanzhou Institute of Chemical Physics) for help in bioassay and for fruitful discussions.

Conflicts of Interest: The authors declare no conflict of interest.

\section{References}

1. Sparks, T.C.; Lorsbach, B.A. Perspectives on the agrochemical industry and agrochemical discovery. Pest. Manag. Sci. 2017, 73, 672-677. [CrossRef] [PubMed]

2. Bardas, G.A.; Veloukas, T.; Koutita, O.; Karaoglanidis, G.S. Multiple resistance of Botrytis cinerea from kiwifruit to SDHIs, QoIs and fungicides of other chemical groups. Pest. Manag. Sci. 2010, 66, 967-973. [CrossRef] [PubMed]

3. Halstead, N.T.; Hoover, C.M.; Arakala, A.; Civitello, D.J.; De Leo, G.A.; Gambhir, M. Agrochemicals increase risk of human schistosomiasis by supporting higher densities of intermediate hosts. Nat. Commun. 2018, 9, 837. [CrossRef] [PubMed]

4. Lamberth, C. Agrochemical lead optimization by scaffold hopping. Pest. Manag. Sci. 2018, 74, 282-292. [CrossRef] [PubMed]

5. Lahouel, M.; Zini, R.; Zellagui, A.; Rhouati, S.; Carrupt, P.A.; Morin, D. Ferulenol specifically inhibits succinate ubiquinone reductase at the level of the ubiquinone cycle. Biochem. Biophys. Res. Commun. 2007, 355, 252-257. [CrossRef] [PubMed] 
6. Dan, W.J.; Tuong, T.M.L.; Wang, D.C.; Li, D.; Zhang, A.L.; Gao, J.M. Natural products as sources of new fungicides $(\mathrm{V})$, Design and synthesis of acetophenone derivatives against phytopathogenic fungi in vitro and in vivo. Bioorg. Med. Chem. Lett. 2018, 28, 2861-2864. [CrossRef] [PubMed]

7. Lamberth, C.; Jeanmart, S.; Luksch, T.; Plant, A. Current challenges and trends in the discovery of agrochemicals. Science 2013, 341, 742-746. [CrossRef] [PubMed]

8. Qasem, J.R. Allelopathy Importance, field application and potential role in pest management. J. Agric. Sci. Technol. 2010, 4, 104-120.

9. de Oliveira, J.R.F.; de Resende, J.T.V.; Maluf, W.R.; Lucini, T.; de Lima, R.B.; de Lima, I.P.; Nardi, C. Trichomes and Allelochemicals in Tomato Genotypes Have Antagonistic Effects Upon Behavior and Biology of Tetranychus urticae. Front. Plant Sci. 2018, 9, 1132. [CrossRef] [PubMed]

10. Kong, C.H.; Xu, X.H.; Zhang, M.; Zhang, S.Z. Allelochemical tricin in rice hull and its aurone isomer against rice seedling rot disease. Pest. Manag. Sci. 2010, 66, 1018-1024. [CrossRef] [PubMed]

11. Nebo, L.; Varela, R.M.; Molinillo, J.M.G.; Sampaio, O.M.; Severino, V.G.P.; Cazal, C.M.; das Graças Fernandes, M.F.; Fernandes, J.B.; Macias, F.A. Phytotoxicity of alkaloids, coumarins and flavonoids isolated from 11 species belonging to the Rutaceae and Meliaceae families. Phytochem. Lett. 2014, 8, $226-232$. [CrossRef]

12. Yan, Z.Q.; Wang, D.D.; Cui, H.Y.; Zhang, D.H.; Sun, Y.H.; Jin, H. Phytotoxicity mechanisms of two coumarin allelochemicals from Stellera chamaejasme in lettuce seedlings. Acta Physiol. Plant. 2016, 38, 248. [CrossRef]

13. Pan, L.; Li, X.Z.; Yan, Z.Q.; Guo, H.R.; Qin, B. Phytotoxicity of umbelliferone and its analogs, Structure-activity relationships and action mechanisms. Plant Physiol. Biochem. 2015, 97, 272-277. [CrossRef] [PubMed]

14. Stevens, B.D.; Bungard, C.J.; Nelson, S.G. Strategies for expanding structural diversity available from olefin isomerization-Claisen rearrangement reactions. J. Org. Chem. 2006, 271, 6397-6402. [CrossRef] [PubMed]

15. Buchman, C.D.; Hurley, T.D. Inhibition of the aldehyde dehydrogenase $1 / 2$ family by psoralen and coumarin derivatives. J. Med. Chem. 2017, 60, 2439-2455. [CrossRef] [PubMed]

16. Chen, Y.; Wang, S.L.; Xu, X.Q.; Liu, X.; Yu, M.Q.; Zhao, S.; Liu, S.C.; Qiu, Y.L.; Zhang, T.; Liu, B.F.; et al. Synthesis and biological investigation of coumarin piperazine (piperidine) derivatives as potential multireceptor atypical antipsychotics. J. Med. Chem. 2013, 56, 4671-4690. [CrossRef] [PubMed]

17. Tucaliuc, R.A.; Cotea, V.V.; Niculaua, M.; Tuchilus, C.; Mantu, D.; Mangalagiu, I.I. New pyridazine-Fluorine derivatives, synthesis, chemistry and biological activity. Part II. Eur. J. Med. Chem. 2013, 67, 367-372. [CrossRef] [PubMed]

18. Berthelot, K.; Estevez, Y.; Quiliano, M.; Baldera-Aguayo, P.A.; Zimic, M.; Pribat, A.; Bakleh, M.E.; Teyssier, E.; Gallusci, P.; Gardrat, C.; et al. HbIDI; SIIDI and EcIDI: A comparative study of isopentenyl diphosphate isomerase activity and structure. Biochimie 2016, 127, 133-143. [CrossRef] [PubMed]

19. Scholte, A.A.; Eubanks, L.M.; Poulter, C.D.; Vederas, J.C. Synthesis and biological activity of isopentenyl diphosphate analogues. Bioorg. Med. Chem. 2004, 12, 763-770. [CrossRef] [PubMed]

20. Zhao, P.L.; Wang, F.; Zhang, M.Z.; Liu, Z.M.; Huang, W.; Yang, G.F. Synthesis, fungicidal, and insecticidal activities of beta-methoxyacrylate-containing $N$-acetyl pyrazoline derivatives. J. Agric. Food Chem. 2008, 56, 10767-10773. [CrossRef] [PubMed]

21. Herms, S.; Seehaus, K.; Koehle, H.; Conrath, U. Astrobilurin fungicide enhances the resistance of tobacco against tobacco mosaic virus and Pseudomonas syringae pv tabaci. Plant Physiol. 2002, 130, 120-127. [CrossRef] [PubMed]

22. Kumagai, H.; Nishida, H.; Imamura, N.; Tomoda, H.; Omura, S.; Bordner, J. The structures of atpenins A4, A5 and B, new antifungal antibiotics produced by Penicillium sp. J. Antibiot. 1990, 43, 1553-1558. [CrossRef] [PubMed]

23. Lv, X.H.; Ren, Z.L.; Liu, P.; Li, B.X.; Li, Q.S.; Chu, M.J.; Cao, H.Q. Design, synthesis and biological evaluation of novel nicotinamide derivatives bearing a substituted pyrazole moiety as potential SDH inhibitors. Pest Manage. Sci. 2017, 73, 1585-1592. [CrossRef] [PubMed]

Sample Availability: Samples of the compounds 1-10 are available from the authors. 